\title{
Overlapping Microarray Profiles of Dentate Gyrus Gene Expression during Development- and Epilepsy-Associated Neurogenesis and Axon Outgrowth
}

\author{
Robert C. Elliott, ${ }^{1}$ Michael F. Miles, ${ }^{2}$ and Daniel H. Lowenstein ${ }^{1}$ \\ ${ }^{1}$ Department of Neurology, Beth Israel Deaconess Medical Center, Boston, Massachusetts 02115, and ${ }^{2}$ Departments of Pharmacology/Toxicology and \\ Neurology, Virginia Commonwealth University, Richmond, Virginia 23298
}

\begin{abstract}
Neurogenesis and axon outgrowth are features shared by normal nervous system development and certain forms of epileptogenesis. This observation has led to the hypothesis that some aspects of normal development and epileptogenesis have common molecular mechanisms. To test this hypothesis, we have used DNA microarray analysis to characterize gene expression in the dentate gyrus and identify genes exhibiting similar patterns of regulation during development and epileptogenesis. Of more than 8000 sequences surveyed, over 600 were regulated during development or epileptogenesis, and 37 of these were either upregulated or downregulated during both processes. In situ hybridization analysis of a subset of these "commonality genes" confirmed the patterns of regulation predicted by the microarray data in most cases and demonstrated various spatial and temporal patterns of commonality gene expression. Of the 25 named commonality genes in which some functional characteristics are known, 11 have been implicated in cell morphology and axon outgrowth or cellular proliferation and fate determination. This enrichment for candidate plasticity-related genes supports the concept that developmental mechanisms contribute to network alterations associated with epileptogenesis and offers a useful strategy for identifying molecules that may play a role in both of these processes.
\end{abstract}

Key words: microarray; development; epilepsy; plasticity; hippocampus; dentate gyrus

\section{Introduction}

Dentate granule cell (DGC) neurogenesis and axon outgrowth are hallmarks of both the normal development of the dentate gyrus and its aberrant reorganization during epileptogenesis. During development, DGC precursors migrate from the embryonic subventricular zone to the hippocampal formation, where they ultimately populate a localized proliferative region along the hilar side of the granule cell layer termed the subgranular zone (SGZ) (Altman and Bayer, 1990). Newly differentiated DGCs begin to extend mossy fiber axons toward the hilus and CA3 regions and eventually migrate away from the SGZ deeper into the granule cell layer. The rate of DGC birth is maximal in the first 2 postnatal weeks, yet the SGZ continues to produce neurons well into adulthood in mammals, although at a reduced rate (Altman and Das, 1965; Bayer, 1980; Kuhn et al., 1996; Eriksson et al., 1998; Gould et al., 1999b). However, in a rat model of human temporal lobe epilepsy, the rate of neurogenesis in the adult rat SGZ is dramatically increased after pilocarpine-induced status epilepticus (SE) (Parent et al., 1997). This increase is evident within 2-3 d after SE and remains highly elevated for 10-14 d before returning to baseline over the next several weeks. Over the same timeframe after SE, DGC mossy fiber axons undergo exten-

\footnotetext{
Received May 17, 2002; revised Dec. 6, 2002; accepted Dec. 11, 2002.

This work was supported by National Institutes of Health Grant R01NS39950 and funding from the State of California for medical research on alcohol and substance abuse through the University of California, San Francisco. We thank Dr. Li Zhang for his helpful discussions regarding S-score statistics and Brian Kruegel and Norb Wilke for their technical contributions.

Correspondence should be addressed to Dr. Daniel Lowenstein, Department of Neurology, Box 0114, University of California, San Francisco, San Francisco, CA 94143-0114. E-mail: dhl@itsa.ucsf.edu.

Copyright $\odot 2003$ Society for Neuroscience $\quad 0270-6474 / 03 / 232218-10 \$ 15.00 / 0$
}

sive remodeling in a process referred to as mossy fiber sprouting (Tauck and Nadler, 1985; Cronin and Dudek, 1988). These axons sprout collaterals that aberrantly cross back through the DGC layer and form synaptic connections with DGC dendrites in the inner molecular layer as well as with interneurons within the DGC layer (Frotscher and Zimmer, 1983; Sloviter, 1992; Okazaki et al., 1995). Mossy fiber sprouting is first visible by Timm staining within $7 \mathrm{~d}$ after SE and continues to increase in intensity for at least 2-3 months. However, studies in which the newborn DGC population is eradicated before SE indicate that both newborn and mature DGCs undergo mossy fiber sprouting (Parent et al., 1999), providing important evidence that epilepsy-associated network rewiring in the adult rat dentate gyrus is not limited to newly born neurons.

The similarities between development- and epilepsyassociated neurogenesis and axon outgrowth raise the question of whether parallel molecular mechanisms underlie these facets of normal and aberrant DGC plasticity. This possibility is supported by numerous reports over the last decade showing that developmentally regulated molecules, such as embryonic neural cell adhesion molecule, tenascin- $\mathrm{C}$, and multiple members of the basic helix-loop-helix (bHLH) family of cell fate determinants, are reexpressed in the dentate after SE (Represa and Ben-Ari, 1997; Pleasure et al., 2000; Elliott et al., 2001). Multiple gene PCR analysis, performed on whole hippocampal homogenates, has identified numerous other genes with similar levels of expression during development and after animal treatment with kainic acid (Chang et al., 2001). On the basis of this evidence, we hypothesize that the molecules guiding DGC neurogenesis and axon out- 
growth during development overlap with those expressed during epileptogenesis.

To test our hypothesis, we have used oligonucleotide microarrays to profile the mRNA levels of more than 8000 genes and expressed sequence tags (ESTs) in developing and epileptogenic rats. Previous investigators have used similar oligonucleotide arrays to characterize regional differences in gene expression (Sandberg et al., 2000; Zirlinger et al., 2001) as well as to analyze changes in gene expression caused by aging or environmental enrichment (Lee et al., 1999; Rampon et al., 2000). However, generally lower expression levels for a more complex variety of genes in the brain, coupled with the high degree of brain cellular heterogeneity, make reliable detection of gene expression changes in specific cell populations of the brain particularly challenging. To address this problem, we (1) focused our microarray analysis on specific timepoints during development and epileptogenesis when neurogenesis and mossy fiber outgrowth are robust, (2) enriched the DGC population in our tissue samples by microdissecting the dentate gyrus away from the rest of the hippocampus, and (3) used an improved analysis algorithm (Sscore) to detect significant changes in gene expression (Zhang et al., 2002). As a result, our microarray analysis identified more than 600 genes (including "named" genes, whose functions have been previously described, and EST sequences) that are regulated during development or epileptogenesis. Screening for those genes similarly regulated during both development and epileptogenes (e.g., upregulated during development and epileptogenesis) resulted in the selection of a subset of genes and ESTs that we collectively refer to as "commonality genes." This group of commonality genes is composed of a large percentage of genes with known or likely functions related to regulation of cell morphology or cell cycle/fate determination.

\section{Materials and Methods}

Induction of SE. All animals were treated according to protocols for animal care established by the University of California, San Francisco (where studies were conducted), and the National Institutes of Health. Adult male Sprague Dawley rats (180-200 gm; Bantin and Kingman, Fremont, CA) were given an injection of atropine methylbromide (5 $\mathrm{mg} / \mathrm{kg}$, i.p.; Sigma, St. Louis, MO) followed $20 \mathrm{~min}$ later by an injection of pilocarpine hydrochloride ( $340 \mathrm{mg} / \mathrm{kg}$, i.p.; Sigma) to induce SE. Seizure activity was monitored behaviorally and terminated with an injection of diazepam (10 mg/kg, i.p.; Elkins-Sinn, Cherry Hill, NJ) after $2 \mathrm{hr}$ of convulsive SE. Only rats that displayed continuous, convulsive seizure activity after pilocarpine treatment were included in these studies. Control rats received the same injections of atropine and diazepam but received saline instead of pilocarpine. Animals were killed $14 \mathrm{~d}$ after SE induction and then dissected for preparation of RNA extracts (as described below) or perfused for in situ hybridization (also as described below).

Animal dissection and preparation of $c R N A$. Animals were deeply anesthetized with isofluorane gas and decapitated, followed by brain removal and hippocampal dissection on an ice-cooled stage. Hippocampal sections $(600 \mu \mathrm{m})$ were cut using a McIlwain tissue chopper and transferred to a Petri dish containing ice-cold PBS for dentate gyrus microdissection. At 20 min after animals were killed, all microdissected tissue was gathered and frozen on dry ice for storage. Subsequently, tissue pooled from 2-3 adult animals or 10-12 postnatal day 3 (P3) animals was homogenized in Trizol reagent (Invitrogen, Carlsbad, CA) using a glass homogenizer (Wheaton, Millville, NJ), and total RNA was isolated after the Trizol protocol. RNA concentration was determined spectrophotometrically, and RNA integrity was confirmed by agarose gel electrophoresis. Equivalent amounts ( $15 \mu \mathrm{g})$ of total RNA derived from each tissue sample were then reverse-transcribed into double-stranded cDNA using Life Technologies Superscript Choice System (Grand Island, NY). Doublestranded cDNA $(0.5-1 \mu \mathrm{g})$ was then used as a template for synthesis of biotin-labeled cRNA using a BioArray HighYield RNA Transcript Labeling Kit (ENZO Diagnostics, Farmingdale, NY) and protocols as recommended by the manufacturer. Labeled cRNA was purified on RNAeasy affinity resin (Qiagen, Mountain View, CA) and quantified by absorbance at $260 \mathrm{~nm}$. Before hybridization, $10 \mu \mathrm{g}$ of cRNA was fragmented randomly to an average size of $50-100$ bases by incubation at $94^{\circ} \mathrm{C}$ for 35 min in $40 \mathrm{~mm}$ Tris-acetate, $\mathrm{pH} 8.1,100 \mathrm{~mm}$ potassium acetate, and $30 \mathrm{~mm}$ magnesium acetate.

Array hybridization and scanning. Labeled cRNA samples were analyzed on Affymetrix Rat Genome U34 Set chip A (Affymetrix, Santa Clara, CA), which represents nearly 7000 named genes and more than 1000 EST sequences. Sample fragmentation, hybridization to the array, and array scanning were performed according to standard Affymetrix protocols and as described previously (Thibault et al., 2000). In brief, aliquots of fragmented cRNA ( $10 \mu \mathrm{g}$ in a $200 \mu \mathrm{l}$ master mix) were hybridized to $\mathrm{U} 34$ GeneChip arrays at $45^{\circ} \mathrm{C}$ for $16 \mathrm{hr}$ in a rotisserie oven under constant rotation $(60 \mathrm{rpm})$. After hybridization, arrays were washed and stained with streptavidin-phycoerythrin (Molecular Probes, Eugene, OR) using an Affymetrix Fluidics Station. Hybridization signals were amplified by incubating arrays at $25^{\circ} \mathrm{C}$ with $3 \mu \mathrm{g} / \mathrm{ml}$ biotinylated goat anti-streptavidin antibody (Vector Laboratories, Burlingame, CA) in the presence of $0.1 \mathrm{mg} / \mathrm{ml}$ normal goat IgG (Sigma) in staining buffer. Arrays were then restained with streptavidin-phycoerythrin, washed with nonstringent buffer, and scanned using a dedicated confocal scanner (Hewlett-Packard, Palo Alto, CA).

Array data analysis. Initial processing of microarray data, including calculation of "average difference" expression intensity levels, was performed using Microarray Suite software (MAS, ver. 4) (Affymetrix). All arrays were normalized by correction to a set value for median total hybridization intensity. Scaling factors for all arrays were between 1.4 and 1.8. Quality of array hybridizations was also assessed by ensuring that the ratio of $3^{\prime}$ - to $5^{\prime}$-end probes for $\beta$-actin and glyceraldehyde-3phosphate dehydrogenase did not exceed 2. Pairwise scattergram comparisons of all arrays showed highly linear behavior across all intensity classes. To qualitatively assess differences between control and experimental samples, scattergrams (see Fig. 1) were generated using genes called "present" in at least one sample by the Affymetrix software decision matrix and having average difference values above 50 .

To generate lists of genes with altered expression patterns, we used an analysis algorithm developed in our laboratories for comparison of two high-density oligonucleotide arrays (Zhang et al., 2002). The S-score analysis determines the likelihood that the hybridization signal for a given gene is different between two arrays. Thus, S-scores were generated between saline versus pilocarpine-treated (SE) groups, P3 versus naive adult animals, and naive versus saline-injected controls, as described in Results. The S-score is the sum of differences between perfect-match/ mismatch probe pairs for the two arrays compared, with weighting for both multiplicative and additive error. $S$-scores are derived to have a mean of zero (no change) with an SD of one. An S-score $\geq 2$ corresponds to $p=0.046$. For generating lists of candidate genes, we prefiltered array data to eliminate genes not having at least one average difference value $>50$ and those having highly variable levels of expression in controls. These highly variable genes likely give rise to false-positives in the developmental and epileptogenic comparisons. These were removed by excluding genes in the top 5\% of S-scores generated for naive versus control (saline-injected) comparisons. These naive versus control comparisons were assumed to identify genes having more biological variability in their expression levels and genes responding to the stress of injections. We then filtered the remaining genes $(n=4823)$ to select those having S-score $\geq 2$ in each of two replicate experiments. Because the S-score is a statistically based comparison score rather than a simple "fold-change," filtering by using an S-score cutoff of $S \geq 2$ is comparable with a $p$ value of $<0.0025$. Qualitatively similar results were obtained when we used the significance analysis of microarrays (Tusher et al., 2001) permutation method to select genes having S-scores significantly different from those of the naive versus control comparison (data not shown).

Hierarchical clustering of S-score analyses was used to provide a graphical display of expression patterns (see Fig. 2). Genes were filtered 
as described above to eliminate genes not expressed and genes with high variability in naive versus control comparisons and genes not showing consistent changes in either the developmental or epileptogenic comparisons. This group of genes $(n=649)$ was then analyzed by hierarchical clustering as described by Eisen et al. (1998).

For ANOVA analysis of developmental or epileptogenic regulation of specific functional groups of genes, we used the Affymetrix NetAffx web site (http://www.affymetrix.com/analysis/index.affx) to identify genes on the rat U34A arrays that contained various keywords in either the gene title or the gene ontology (GO) descriptors. These lists of genes were filtered manually to eliminate obvious erroneous entries. Finally, the gene lists were filtered to remove genes not expressed or with high variability in the naive-control comparisons as described above. S-score data from the developmental (P3 vs naive adult), epileptogenesis (pilocarpine-treated vs saline-treated), and control (naive vs saline-control) comparisons were then analyzed by ANOVA for each gene list. Scheffe's post hoc analysis was used to identify significant $(p<0.05)$ pairwise differences (see Table 2).

Nonradioactive in situ hybridization. Animals were given an anesthetic overdose of pentobarbital and transcardially perfused with $300 \mathrm{ml}$ of a $4 \%$ paraformaldehyde solution in $\mathrm{PBS}$ at $\mathrm{pH}$ 7.4. Frozen coronal sections $(20 \mu \mathrm{m})$ were cut and melted onto Superfrost Plus slides (Fisher Scientific, Pittsburgh, PA). Nonradioactive in situ hybridization was performed essentially as described previously (Elliott et al., 2001). Briefly, sections were pretreated with Proteinase K for 5 min before prehybridization at $65^{\circ} \mathrm{C}$ for $3 \mathrm{hr}$ in a solution containing yeast tRNA, Denhardt's solution, and $50 \%$ formamide. After prehybridization, sections were incubated overnight with digoxigenin-labeled probes (see below) at a final concentration of $1 \mathrm{ng} / \mu \mathrm{l}$. Slides were washed the next day at high stringency and incubated with sheep anti-digoxigenin Fab fragments conjugated to alkaline phosphatase (diluted 1:2000; Roche Molecular Biochemicals, Indianapolis, IN) for $2-3 \mathrm{hr}$ at room temperature. After washes, the slides were incubated in buffer containing nitrobluetetrazolium and 5-bromo-4-chloro-3-indoyl phosphate (Roche Molecular Biochemicals) until developed. In situ data were evaluated qualitatively, on the basis of the comparison of mRNA expression patterns in comparable hippocampal sections from batch-processed control and experimental animals. Each in situ hybridization analysis was repeated independently a minimum of three times on tissue sections from different animals.

Preparation of digoxygenin-labeled RNA probes. DNA templates used for generating in situ riboprobes corresponding to selected commonality genes (including ESTs) were amplified by PCR from neonatal or adult rat hippocampal cDNA libraries. Using the Lig n' Scribe protocol (Ambion, Austin, TX), an adapter containing a T7 polymerase start site was ligated to the purified amplicon, followed by a second round of PCR to generate both sense and antisense templates. Sense and antisense digoxygeninlabeled riboprobes were transcribed from their respective DNA templates using the Genius RNA labeling kit (Roche Molecular Biochemicals) and purified with Chromaspin-100 columns packed in DEPC water (Clontech Laboratories, Palo Alto, CA). Probe concentrations were determined spectrophotometrically, and probe integrity was verified by PAGE.

\section{Results}

\section{Generation of microarray gene expression data}

Changes in DGC gene expression during development and epileptogenesis were broadly evaluated using oligonucleotide-based DNA microarrays representing close to 7000 named genes and more than 1000 ESTs. These arrays were used to probe labeled cRNA derived from microdissected dentate gyrus tissue samples from experimental and control animals. In our developmental analysis, microarray gene expression data from P3 rats were compared with naive adult animals. In our epileptogenesis analysis, microarray data from $14 \mathrm{~d}$ post-SE rats were compared with saline-treated adult animals. To increase reliability, microdissected dentate gyrus tissue from two or more animals was pooled for each sample, and each microarray evaluation was performed twice with RNA samples derived independently. Comparisons of

\section{control}

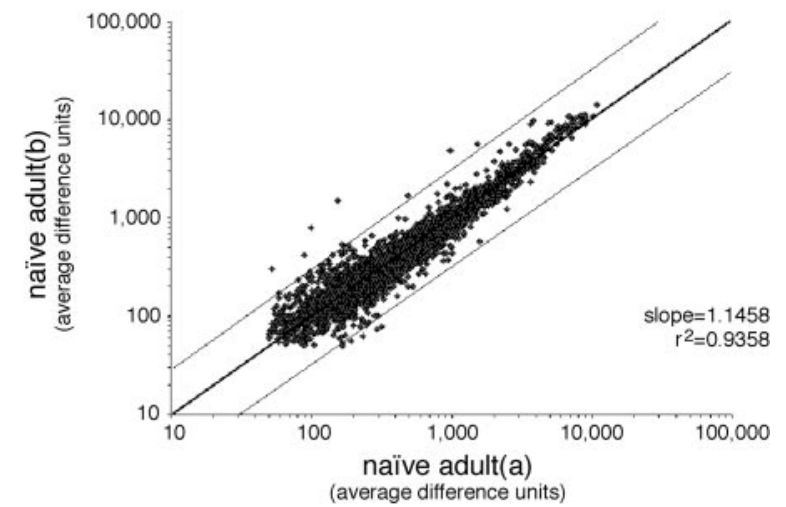

development

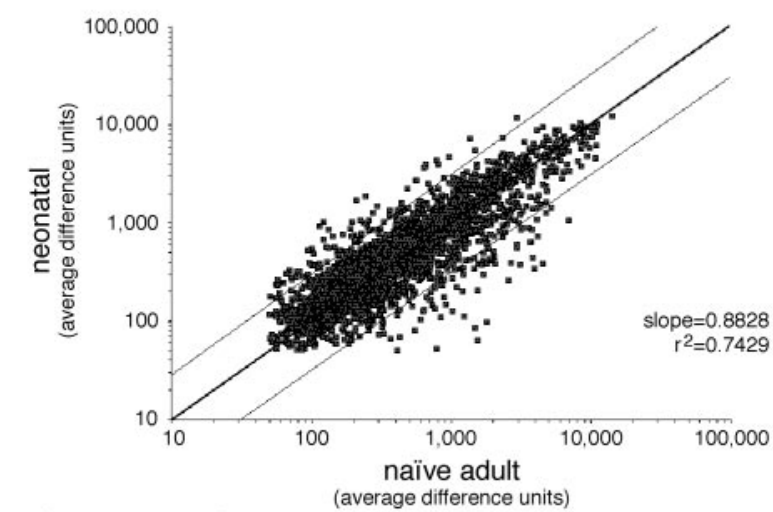

epileptogenesis

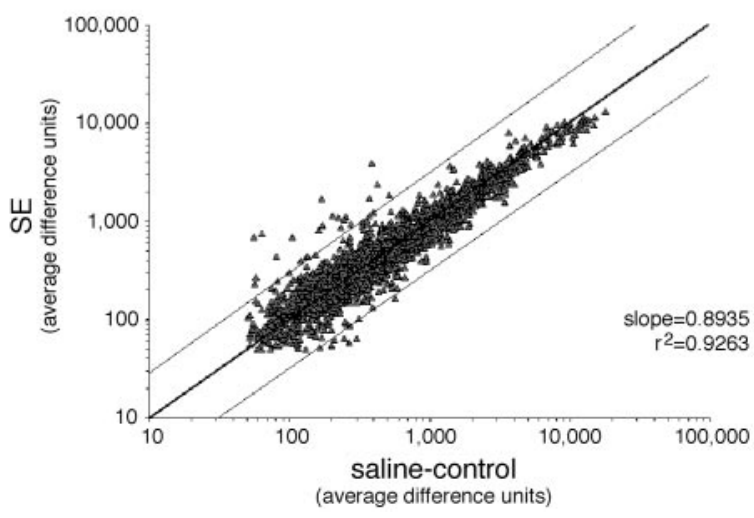

Figure 1. Scattergram analysis of microarray data. Average difference (AD) measures of gene expression for each of the 2680 detectable sequences on the microarray were plotted to yield scattergrams similar to the representative examples shown here. The high degree of correlation of $A D$ values from two independent naive adult dentate gyrus cRNA samples reflects the accuracy of gene expression data derived from the DNA microarrays (top panel). Greater scattering in development and epileptogenesis samples is indicative of differential gene expression during those processes (middle and bottom panels). Light lines on either side of the darker line of equality indicate twofold changes in mRNA levels.

gene expression data for duplicate samples demonstrated a high degree of hybridization signal reproducibility. In a representative comparison of two independently prepared and hybridized naive adult samples, the hybridizations show very linear slope (slope $=$ $1.1458 ; r^{2}=0.9358$ ), with very few of the 2680 genes expressed on both arrays having a more than twofold difference in expression (Fig. 1, top panel). In contrast, developmental (Fig. 1, middle panel) $\left(r^{2}=0.7429\right)$ and epileptogenic (Fig. 1, bottom panel) $\left(r^{2}=0.9263\right)$ comparisons showed greater variability, reflecting 
the relative changes in gene expression. In addition to illustrating the reliability of our oligonucleotide microarrays, these figures also suggest, as expected, that a greater degree of transcriptional regulation occurs during development than during epileptogenesis.

To more accurately identify genes significantly regulated during development or epileptogenesis, we used the recently reported S-score method for all subsequent analyses of microarray data (Zhang et al., 2002). In brief, this algorithm measures the likelihood that a difference in expression of a given gene between two samples is significant and better utilizes the statistical power of having 20 probe pairs per gene on the microarray by adjusting the weighting of each individual probe pair contribution on the basis of the signal strength above background noise. S-scores are normalized to have a mean of 0 and a SD of 1 . Thus, using criteria of having an S-score $\geq 2$ (for upregulated genes) or S-score $\leq 2$ (for downregulated genes) in each of two replicate experiments should result in a false-positive rate of $0.25 \%$, or $\sim 20$ of 8000 genes assayed. Following these criteria, and after removing genes that showed hypervariable expression between control samples, genes that were significantly upregulated or downregulated during development or epileptogenesis were tabulated. The number of genes exhibiting altered gene expression during development was significantly higher than during epileptogenesis, as suggested by the scattergram analysis above, with 509 genes/ESTs being upregulated or downregulated during development and 129 genes/ESTs regulated during epileptogenesis. When these array results were analyzed by hierarchical clustering (Eisen et al., 1998) to identify tentative correlations in expression patterns, prominent groups of developmentally regulated genes were identified that contained smaller clusters of genes similarly regulated during epileptogenesis (Fig. 2). This indicates some degree of overlap between development-regulated genes and epileptogenesisregulated genes. Interestingly, the cluster diagram also indicates that there is significantly more overlap between development and epileptogenesis in genes that are downregulated by both processes.

\section{Functional distributions of genes regulated during development and epileptogenesis}

To assess functional similarities of clustered genes, we categorized each regulated gene into 1 of 11 functional groups on the basis of its previous description in the literature. Of the 638 regulated sequences identified in our S-score analysis, 405 represented named genes and 233 were ESTs. In general, genes regulated during development or epileptogenesis encompassed a wide range of function, including immediate-early response genes, calcium homeostasis genes, cell signaling genes, cell cycle and fate determination genes, morphology and structure genes, and injury/survival genes (Fig. 3). However, when we focused on genes upregulated during development, we found that genes associated with metabolism were twice as numerous as genes from any other category (Fig. 3a). Similarly, when focusing on genes upregulated during epileptogenesis, a much higher percentage of genes involved in injury response or cell survival were identified (Fig. 3a). In contrast, the distributions of genes downregulated during development and epileptogenesis were more uniform, with metabolic genes and signaling genes only slightly more prevalent during development. There was also a modest predominance of metabolic, morphology, and extracellular signaling genes downregulated during epileptogenesis (Fig. $3 b$ ).

We next searched our dataset of regulated genes for those that are similarly upregulated or downregulated during both development and epileptogenesis. We identified 14 named genes and ESTs that are upregulated during both development and epileptogenesis, and 23

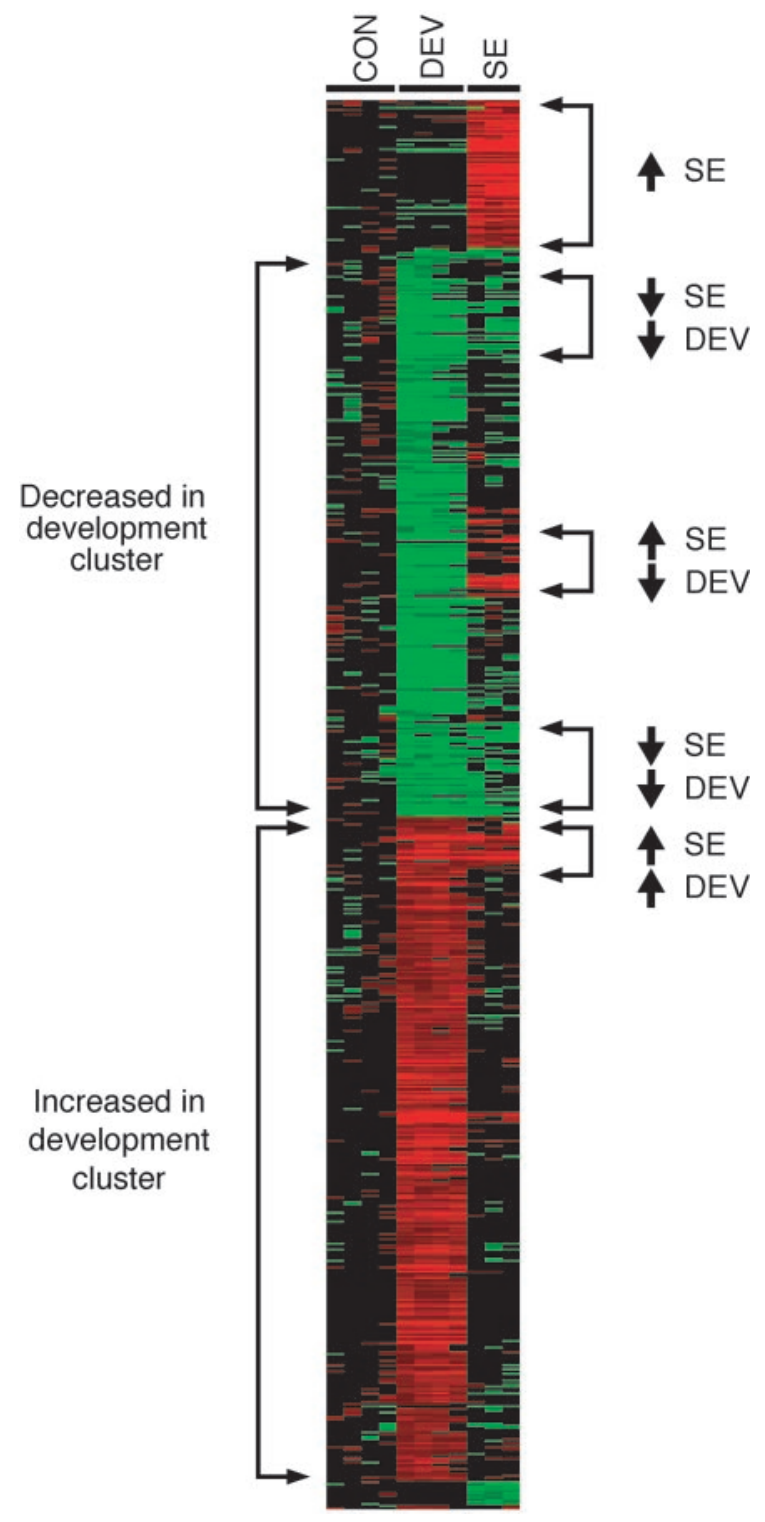

Figure 2. Clustergraph analysis of gene regulation. A hierarchical clustering analysis of $S$-score gene expression data from three epileptogenic comparisons (SE lanes) and four developmental comparisons (DEV lanes) along with four control comparisons (CON lanes) is depicted. Red bands indicate increased gene expression after SE or during development, and green bands indicate decreased gene expression. A large cluster of genes downregulated during both development and epileptogenesis is most apparent; however, smaller subclusters of genes dissimilarly regulated are also evident, as is a separate cluster of genes upregulated during epileptogenesis that are unaffected during development. In CON lanes, red or green bands indicate increased or decreased gene expression between two identical samples.

named genes and ESTs that are downregulated during both processes, for a total of 37 "commonality genes." Using the functional categories described above, we found that the distribution of categories within our list of commonality genes is strikingly different from the functional distributions of development- or epileptogenesisregulated genes. In contrast to the distributions of genes upregulated during development or epileptogenesis separately, upregulated commonality genes consist primarily of morphology genes and cell cycle/fate genes, with relatively few metabolic or injury/survival genes (Fig. $3 a$ ). The largest category of downregulated commonality genes is involved in calcium homeostasis, a category that was a minor constituent of the initial lists of downregulated genes (Fig. $3 b$ ). In addition, a significant number of EST sequences were also identified 
in our commonality screening (Table 1) (the complete lists of development- and epileptogenesis-regulated genes identified in this study are available at http://home. caregroup.org/templatesnew/departments/ BID/neurology-bpe/uploaded_documents/ RCE-JNeurosciMarch03.

Other studies have indicated that nonbiased interrogation of array data by functional group assessment can provide insight into biological mechanisms (Mirnics et al., 2000; Middleton et al., 2002). Therefore, we conducted an ANOVA analysis of S-score data from 722 genes on our microarrays that could be grouped into 15 functional gene clusters on the basis of annotations from UniGene or the GO databases. S-score data across multiple developmental, epileptogenic, and control comparisons were analyzed for each of these clusters, which overlap in varying degrees with the functional categories that we used previously. Despite the greater functional heterogeneity of the genes in these gene groups, seven different clusters demonstrated significant differences $(p<$ 0.05) between developmental, epileptogenic, and control S-scores, including morphology-related genes, defense/ stress-related genes, and calcium-related genes (Table 2). Gene clusters that did not show significant differences included transcription-related, metabolism-related, and cell cycle-related clusters.

\section{Independent verification of commonality genes}

Literature searches for each of the 25 named commonality genes revealed that 12 of them have been shown previously to be developmentally regulated in a manner that is consistent with our array data. Of these 12 genes, 3 have also been shown to be regulated in the hippocampus after seizures or SE in a manner that was also consistent with our array findings (Table 1). One of these previously described commonality genes, neuropeptide $\mathrm{Y}$, is an endogenous regulator of neuronal excitability that is believed to have anticonvulsant properties (Baraban et al., 1997; Woldbye et al., 1997). In agreement with our data, previous reports have shown that NPY expression is abundant at birth and decreases during adulthood, yet is markedly increased in DGCs after seizures (Kowalski et al., 1992; Lurton and Cavalheiro, 1997).

To identify the cellular location of expression changes and further assess the accuracy of our microarray analysis, we qualitatively measured changes in mRNA levels of 17 commonality genes ( 3 other genes showed no detectable signal) during development and epileptogenesis using a nonradioactive in situ hybridization procedure. Characterization of nine upregulated commonality genes indicated that seven showed elevated levels of mRNA in the dentate gyrus of P3 and $14 \mathrm{~d}$ post-SE animals as compared with adult controls (Fig. 4). Eight downregulated com- a) Upregulated commonality genes

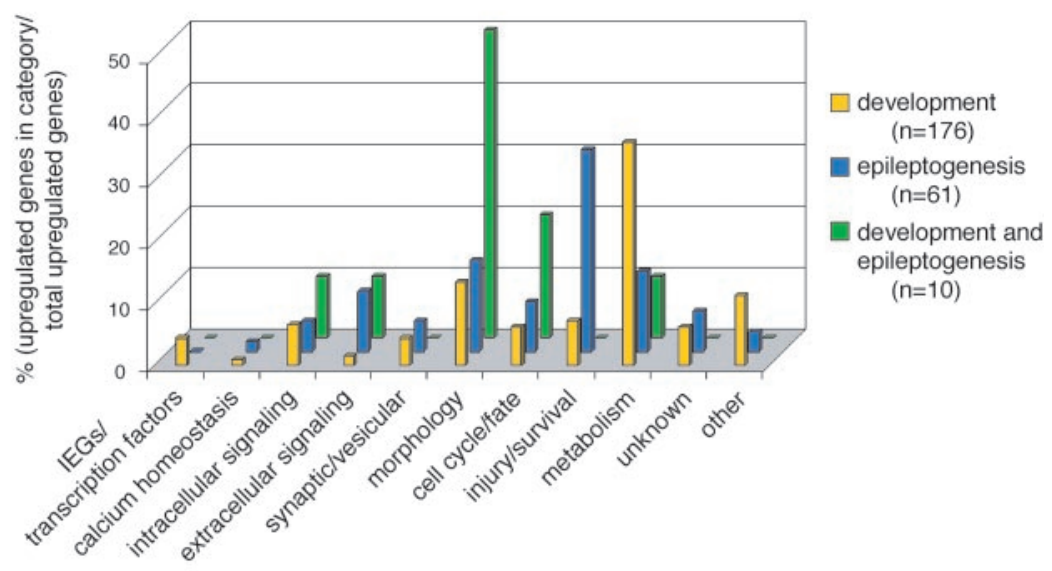

b) Downregulated commonality genes

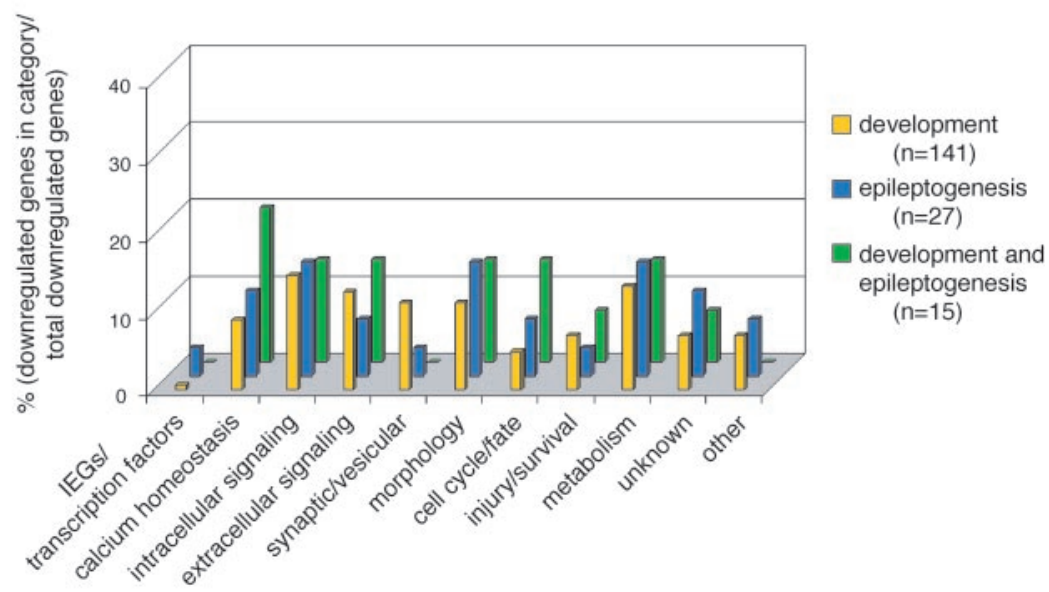

Figure 3. Functional distribution of genes regulated during development and epileptogenesis. Significantly regulated genes were categorized into 1 of 11 functional groups on the basis of their previous descriptions in the literature; EST sequences were not icant fraction of genes upregulated during development ( yellow bars), and injury/survival-related genes are highly upregulated fter SE (blue bars). $b$, Genes downregulated during development or epileptogenesis are distributed more evenly across numerous fategories, with a slight predominance of metabolic and signaling genes during development ( yellow bars) and cium homeostasis-related, and cell cycle/fate-related genes are the categories most frequently regulated during both development and epileptogenesis ( $a, b$, green bars).

monality genes were similarly analyzed, of which five showed mRNA levels that decreased during development and epileptogenesis (Fig. 4). All false-positives from both categories showed developmental expression that was in agreement with the microarray evaluation but failed to exhibit appropriately directed changes in gene expression after SE (including two instances in which in situ data were inconclusive). The apparently greater difficulty in identifying epileptogenesis-regulated genes is likely attributable to the variable extent of cell injury and death after SE (caused by both the prolonged seizures and occasional ischemiarelated damage).

\section{Spatial patterns of commonality gene expression}

In addition to assessing the accuracy of our microarray analysis, our in situ hybridization studies showed overlapping yet independent patterns of commonality gene expression and regulation. Commonality genes upregulated during development, such 
Table 1. Summary of commonality gene expression data

\begin{tabular}{|c|c|c|c|c|c|}
\hline \multicolumn{3}{|l|}{ Upregulated commonality genes } & \multicolumn{3}{|l|}{ Downregulated commonality genes } \\
\hline & Literature & In situ & & Literature & In situ \\
\hline Sox11 & $\mathrm{D}$ & $D, E$ & Hippocalcin & $\mathrm{D}$ & $D, E$ \\
\hline$\alpha$-prothymosin & & $D, \pm$ & NVP-2 & & \\
\hline Protein kinase C receptor & & $D_{1}-$ & PMCA-3 & & \\
\hline Neuropeptide Y & $D, E$ & & Inositol 1,4,5-triphosphate receptor & & \\
\hline Thymosin $\beta-10$ & D & $D, E$ & $\begin{array}{l}\text { Type II calcium/calmodulin-dependent protein } \\
\text { kinase } \beta \text { subunit }\end{array}$ & & \\
\hline CD9 & & $D, E$ & & & \\
\hline CD24 & D & $D, E$ & GABA-A receptor $\Delta$ subunit & $D, E$ & \\
\hline$\beta$ tubulin T $\beta$-15 & $D$ & & Prodynorphin & $D$ & \\
\hline Vimentin & $D, E$ & $D, E$ & Spinophilin & & $\mathrm{D},-$ \\
\hline Fatty acid binding protein & D & & $\begin{array}{l}\text { Dendrin } \\
\text { GAS-7 }\end{array}$ & & $D, \pm$ \\
\hline Expressed sequence tags & & & R-esp1 & & $D, E$ \\
\hline AA944422 & & $D, E$ & PDK2 & D & \\
\hline Al104524 & & $D, E$ & D-dopachrome tautomerase & & \\
\hline \multirow[t]{8}{*}{ Al231807 } & & & Neural membrane protein 35 & D & $\mathrm{D}, \mathrm{E}$ \\
\hline & & & Expressed sequence tags & & \\
\hline & & & AA858621 & & $D, E$ \\
\hline & & & AA859663 & & $\mathrm{D},-$ \\
\hline & & & AA892462 & & $\mathrm{D}, \mathrm{E}$ \\
\hline & & & AA892570 & & \\
\hline & & & AA894317 & & \\
\hline & & & H31144 & & \\
\hline
\end{tabular}

All upregulated and downregulated commonality genes, including named genes and EST sequences, are listed. Literature searches were performed on each named commonality gene to identify previous reports of regulation during development or after chronic seizures. The results of these literature searches are shown in the column labeled Literature, with D indicating previous evidence of developmental regulation that is in agreement with our microarray data and E indicating previous evidence of seizure- or epilepsy-associated regulation that is in agreement with our microarray data. In no instance did our microarray data contradict previous observations in the literature. It is important to note that the experimental conditions in these reports only approximated those used presently (for instance, in many cases kainic acid, instead of pilocarpine, may have been used as a chemoconvulsant, or expression was analyzed at slightly different timepoints). More direct validation of our microarray data was provided by in situ hybridization analysis of commonality gene expression (see Results; Fig. 4). The results of this analysis are compiled in the right column, with D representing positive in situ evidence of developmental regulation that is in agreement with our microarray data, and E representing positive in situ evidence of epileptogenesis-associated regulation that is in agreement with our microarray data. Minus symbols ( - ) indicate in situ evidence that was contrary to our microarray data, i.e., no regulation was observed or regulation was in the opposite direction to what the microarray data indicated. Plus/minus symbols ( \pm ) indicate that variability between experiments was too great to determine definitively whether regulation agreed or disagreed with the microarray data.

as the actin-sequestering peptide thymosin $\beta-10$ or the transcription factor Sox11, were expressed diffusely throughout the dentate gyrus and hilar region, reflecting the ongoing migration and differentiation of the DGC population at this stage (Fig. 4). Not surprisingly, the upregulated commonality genes typically exhibited prominent expression in the neurogenic subgranular zone of the adult, with variably lower levels of expression throughout the rest of the dentate gyrus. These patterns contrasted with the more uniform expression throughout the dentate gyrus that was frequently seen in downregulated commonality genes, such as the cell fate determinant $R$-esp-1 and the uncharacterized neural membrane protein 35 (Fig. 4, bottom). These relative patterns of expression were retained for the most part after SE, with proportionate increases or decreases in the SGZ and the rest of the dentate compared with the normal adult. However, there were notable exceptions. For instance, thymosin $\beta-10$, which was preferentially expressed in the SGZ of the normal adult dentate gyrus, showed a substantially greater increase in the more mature region of the DGC layer after SE (Fig. 4).

\section{Temporal patterns of commonality gene expression}

We next sought to determine more about the time courses and anatomical patterns of commonality gene expression after SE, focusing on those commonality genes with functions related to morphology, cell cycle/fate, or calcium homeostasis. In situ hybridization analysis was performed at 3, 7, 14, and $28 \mathrm{~d}$ after SE, a timeframe that encompasses the rise, peak, and return to baseline levels of neurogenesis as well as the onset and establishment of detectable mossy fiber outgrowth. Initial priority for this analysis was given to those genes that exhibited the most dramatic changes in expression in our previous studies, such as CD24, that codes for a cell-surface glycoprotein that interacts with L1 and integrin to potentially influence cell interactions (Kleene et al., 2001), or those that showed particularly interesting patterns of altered expression within the dentate gyrus, such as hippocalcin, that codes for a neuronal calcium sensor protein. Results from these experiments illustrated that, similar to their different spatial patterns of expression, the temporal profiles of commonality gene expression are also varied, with changes in gene expression occurring either acutely or over a more prolonged period. For example, mRNA coding for CD24 shows its greatest increase at $7 \mathrm{~d}$ after SE, followed by a gradual decline toward control levels over the following 3 weeks (Fig. 5). Expression of CD24 occurs primarily in the SGZ throughout this time course, in agreement with its previously described expression in newly born neurons (Shirasawa et al., 1993). In contrast, expression of hippocalcin mRNA, which is seen throughout the DGC layer in the normal adult, is decreased to the greatest extent at $14 \mathrm{~d}$ after SE and only partially rebounds by $28 \mathrm{~d}$ after SE. Moreover, this decrease is most apparent in the outer region of the granule cell layer, a region that is populated by more mature DGCs (Fig. 5).

\section{Discussion}

In this report, we have explored the hypothesis that common molecular mechanisms underlie features of DGC neurogenesis and axon outgrowth that are shared between development and epileptogenesis. Although evidence supporting this hypothesis has for the most part been generated one gene at a time, the advancement of DNA microarray technologies has opened the way for testing of this hypothesis on a far broader scale. Our 
Table 2. ANOVA analysis of $\mathrm{GO}$-annotated gene clusters

\begin{tabular}{|c|c|c|c|}
\hline Gene group & Treatment & $\begin{array}{l}\text { S-score } \\
\text { (mean }+ \text { SE) }\end{array}$ & Scheffe's post hoc \\
\hline \multirow{3}{*}{$\begin{array}{l}\text { Calcium }{ }^{\#} \\
\qquad(n=62)\end{array}$} & Epil & $-0.65 \pm 0.12$ & $p=0.013$ (vs Con) \\
\hline & Dev & $-1.07 \pm 0.18$ & $p<0.0001$ (vs Con) \\
\hline & Con & $-0.16 \pm 0.09$ & \\
\hline \multirow{3}{*}{$\begin{array}{l}\text { Cell adhesion }{ }^{++} \\
\quad(n=29)\end{array}$} & Epil & $0.07 \pm 0.13$ & $p=0.005$ (vs Dev) \\
\hline & Dev & $0.90 \pm 0.29$ & $p=0.007$ (vs Con) \\
\hline & Con & $0.10 \pm 0.12$ & \\
\hline \multirow{3}{*}{$\begin{array}{l}\text { Channels }^{\#} \\
\qquad(n=73)\end{array}$} & Epil & $-0.05 \pm 0.08$ & $p=0.0007$ (vs Dev) \\
\hline & Dev & $-0.50 \pm 0.09$ & $p<0.0001$ (vs Con) \\
\hline & Con & $0.03 \pm 0.07$ & \\
\hline \multirow{3}{*}{$\begin{array}{l}\text { Defense/stress* } \\
\quad(n=20)\end{array}$} & Epil & $1.39 \pm 0.41$ & $p=0.042$ (vs Con) \\
\hline & Dev & $1.11 \pm 0.36$ & \\
\hline & Con & $0.30 \pm 0.13$ & \\
\hline \multirow{3}{*}{$\begin{array}{l}\text { Neurotransmitter }{ }^{\#} \\
\text { receptors } \\
(n=68)\end{array}$} & Epil & $-0.43 \pm 0.10$ & $p=0.0029$ (vs Con), \\
\hline & Dev & $-1.14 \pm 0.17$ & $p=0.0005$ (vs Dev) \\
\hline & Con & $0.12 \pm 0.11$ & $p<0.001$ (vs Con) \\
\hline \multirow{3}{*}{$\begin{array}{l}\text { Ribosomal }^{\#} \\
\qquad(n=112)\end{array}$} & Epil & $0.20 \pm 0.09$ & $p=0.027$ (vs Con), \\
\hline & Dev & $2.05 \pm 0.10$ & $p<0.0001$ (vs Dev) \\
\hline & Con & $-0.16 \pm 0.11$ & $p<0.0001$ (vs Con) \\
\hline \multirow{3}{*}{$\begin{array}{l}\text { Synaptic/vesicle }{ }^{++} \\
\qquad(n=35)\end{array}$} & Epil & $0.31 \pm 0.19$ & $p=0.0013$ (vs Dev) \\
\hline & Dev & $-0.73 \pm 0.26$ & $p=0.0128$ (vs Con) \\
\hline & Con & $0.10 \pm 0.14$ & \\
\hline
\end{tabular}

Seven hundred twenty-two genes represented on the Affymetrix microarray, previously annotated by the Genome Organization (GO) and categorized into 15 different functional clusters, were analyzed by ANOVA. S-score values of repeated developmental (Dev), epileptogenic (Epil), or control (Con) comparisons were analyzed to identify clusters with significant differences among the three groups $(p<0.05)$ as shown above ( $n=$ number of genes in cluster). Scheffe's post hoc analysis indicates significant intragroup differences and respective $p$ values. Other clusters analyzed that did not demonstrate significant differences were apoptosis $(n=25)$, cell cycle $(n=20)$, differentiation $(n=18)$, G-protein signaling $(n=15)$, metabolism $(n=72)$, neuropeptides $(n=10)$, signaling $(n=93)$, and transcription $(n=70)$.

ANOVA: ${ }^{\#} p<0.0005,{ }^{++} p<0.005,{ }^{*} p<0.05$.

microarray analysis has resulted in the identification of more than 600 genes (including named genes and EST sequences) that appear to be regulated during development or epileptogenesis. During development of the dentate gyrus in the rat, the numbers of genes upregulated and downregulated are comparable, in agreement with what has been found in microarray studies of mouse hippocampal development (Mody et al., 2001). In contrast, most of the genes are upregulated during epileptogenesis, similar to what has been reported for acute changes in kainateinduced gene expression (Hevroni et al., 1998). Moreover, we found a predominant upregulation of metabolic genes during development, which is consistent with the high level of biosynthesis during this period. During epileptogenesis, the upregulation of numerous genes involved with the injury response or cell survival is not surprising given the damage induced by SE (Sloviter, 1996; Houser, 1999; Lowenstein, 2001). Although these profiles of gene regulation are congruous with the expected cellular demands and alterations of development and epileptogenesis, it is also possible that they merely result from a higher representation of metabolic and injury/survival genes on the microarray. Because all 7000 named genes on the array have not yet been categorized functionally, we are unable to answer this question directly. However, the lack of any one outstanding category of gene function in the list of genes downregulated during development or epileptogenesis supports the likelihood that the distributions of regulated genes that we have identified reflect actual cellular profiles of DGC gene expression.

Commonality screening of the 509 genes regulated during development with the 129 genes regulated during epileptogenesis identified 37 genes shared between the two groups. We found an extremely interesting shift in the functional composition of the roster of commonality genes as compared with the initial source

\section{Upregulated commonality genes}

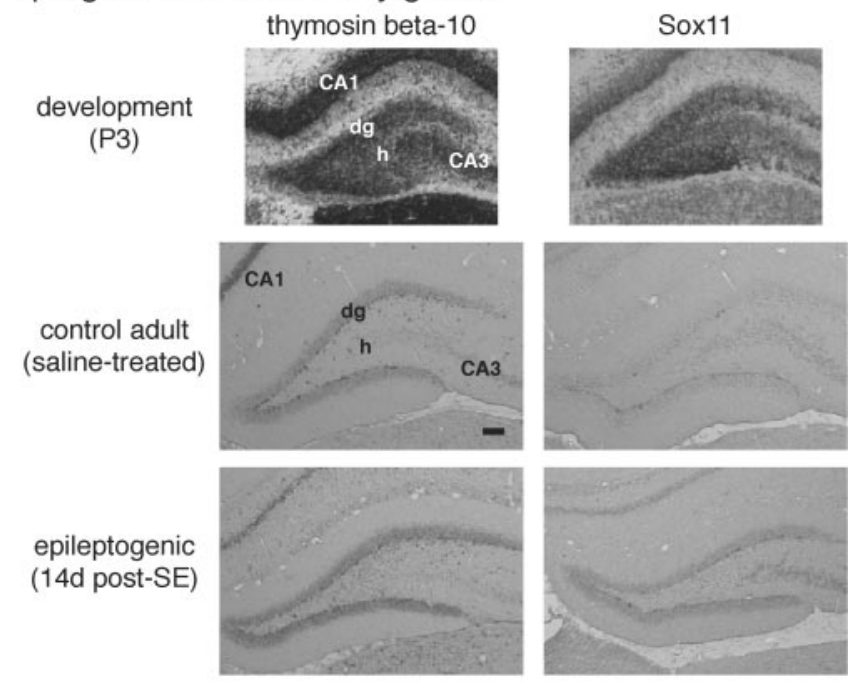

\section{Downregulated commonality genes}

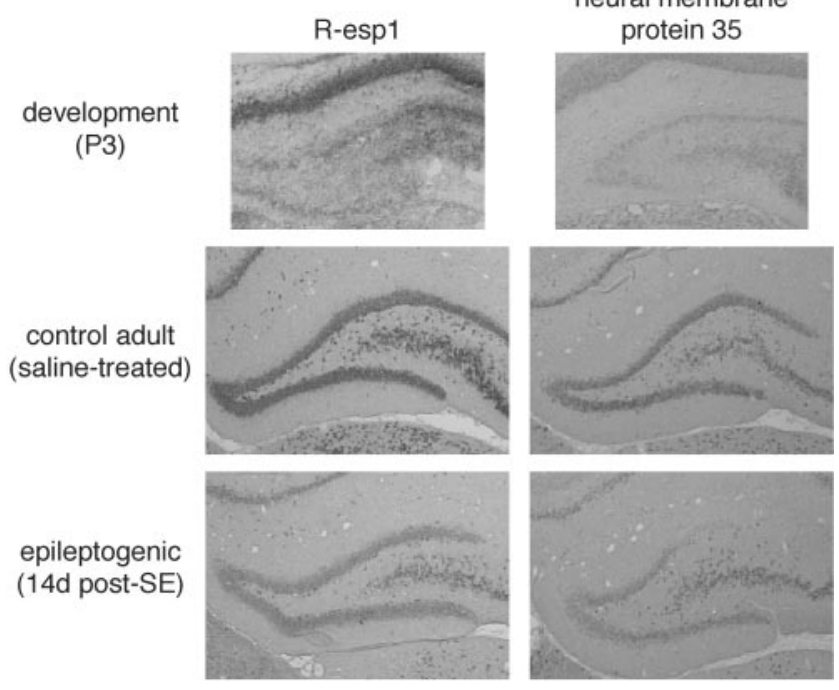

Figure 4. In situ characterization of commonality gene expression in developing, normal adult, and epileptogenic adult dentate gyrus. In situ mRNA analysis of 17 representative commonality genes (including 8 EST clusters) from multiple functional categories was performed to characterize cellular patterns of gene expression and verify DNA microarray regulatory data. Examples of "true positive" upregulated commonality genes, with higher levels of expression in dentate gyrus tissue sections from P3 and $14 \mathrm{~d}$ post-SE animals as compared with adult control, are shown in top half of figure. Note diffuse labeling for thymosin $\beta-10$ and Sox 11 mRNAs throughout developing dentate gyrus at P3 that is accentuated in the adult DGC layer, with the most prominent signal in the neurogenic SGZ. Examples of true positive downregulated commonality genes, with mRNA levels that are downregulated during development and epileptogenesis, are shown in the bottom half of the figure. At P3, R-esp1 and neural membrane protein 35 expression are relatively light and more limited within the formative DGC layer, most likely in more mature cells, in keeping with the greater levels of expression in the adult. The degree of upregulation or downregulation of commonality gene expression during epileptogenesis ranged from slight (e.g., Sox11, top) to large (e.g., R-esp 1, bottom). CA1, Corpus ammon 1 pyramidal cell layer; $C A 3$, corpus ammon 3 pyramidal cell layer; $d g$, dentate gyrus; $h$, hilus. Scale bar, $100 \mu \mathrm{m}$.

lists. In the group of genes that are upregulated during development and epileptogenesis, there is a marked increase in the relative numbers of morphology-related genes and cell cycle/fate genes. Morphology-related commonality gene products include extracellular glycoproteins, such as CD9 and CD24, as well as intracellular molecules involved in cytoskeletal structure, such as 
cd24

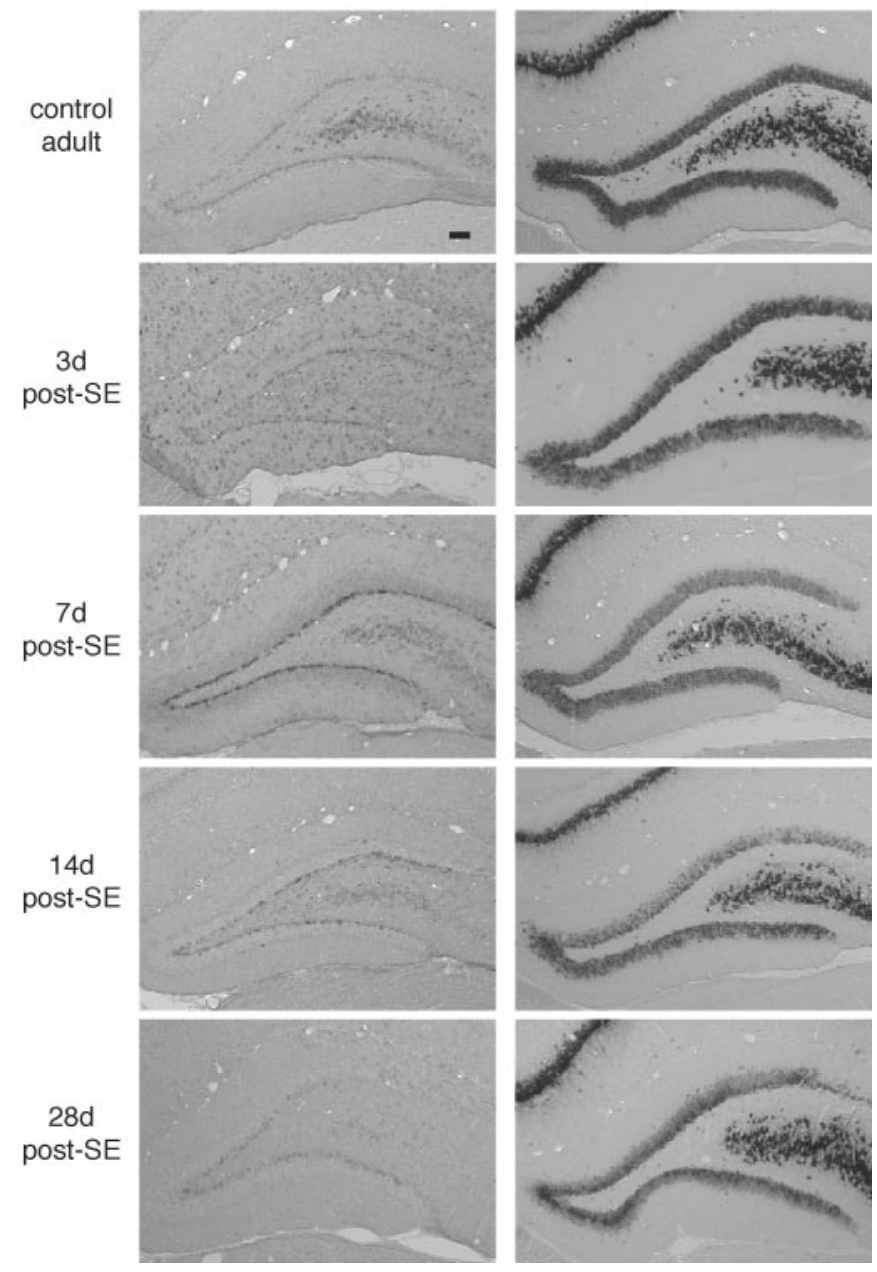

Figure 5. In situ analysis of commonality gene expression over $28 \mathrm{~d}$ time course of epileptogenesis. In situ analysis of selected commonality genes was conducted over a broader time course of epileptogenesis. Various spatial and temporal patterns of gene expression and regulation were observed. For example, CD24 mRNA was prominently expressed in the SGZ of the dentate gyrus (other than a more global induction at $3 \mathrm{~d}$ post-SE that appears to be in glia and is relatively short-lived) and increased acutely after SE (left), whereas hippocalcin mRNA was more broadly expressed throughout the dentate gyrus and exhibited a more prolonged decrease that was greatest in the more mature regions of the cell layer (right). Scale bar, $100 \mu \mathrm{m}$.

the actin-binding protein thymosin $\beta$-10, and $\mathrm{T} \beta$-15, a developmentally regulated isoform of $\beta$-tubulin (Ginzburg et al., 1985). Upregulated commonality gene products associated with cell cycle/fate determination include Sox11, a member of the Sox gene family of transcription factors with diverse roles in vertebrate development and differentiation (Pevny et al., 1998; Wegner, 1999), and prothymosin $\alpha$, a nuclear protein linked to cell proliferation that is transcriptionally regulated by c-myc (Gaubatz et al., 1994). In light of the fact that our microarrays represent an estimated $25 \%$ or less of the entire rat genome, and given the limitations of our current experimental design (see below), we expect that there are many other commonality genes to be identified. At this point, however, the enrichment of genes involved in cell morphology and structure or cell cycle and fate determination in our list of commonality genes represents a shift in gene representation that is predicted by our hypothesis of parallel DGC gene expression underlying developmental and epileptogenic plasticity.

Although we have focused on genes that are most likely to be directly involved in neurogenesis and axon outgrowth, the unbiased nature of our commonality screening strategy has also allowed us to identify other genes similarly regulated during development and epileptogenesis that we had not anticipated previously. For example, the category of calcium homeostasisrelated genes, whereas not as dramatically changed as the morphology- and cell cycle/fate-associated genes, showed a doubling in relative numbers in the list of downregulated commonality genes. These gene products include two neuronal calcium sensor proteins, NVP-2 and hippocalcin, which are involved in calcium-dependent regulation of multiple signal transduction cascades (Braunewell and Gundelfinger, 1999), as well as the calcium-binding protein calbindin. Although not obvious, the enrichment of this category in the list of commonality genes is not surprising given the multifaceted role of calcium in a diverse variety of signaling pathways from transcriptional activation to synaptic strengthening. Long-term alterations in the regulation of intracellular calcium levels in pyramidal neurons after pilocarpine-induced epileptogenesis have been reported previously (Raza et al., 2001), including a decreased ability to restore glutamate-induced calcium levels to baseline. Cytoplasmic calcium has also been shown to mediate the ability of electrical activity to influence growth cone guidance by diffusible factors (Ming et al., 2001), and filopodial calcium transients promote substrate-dependent growth cone turning (Gomez et al., 2001). Interestingly, components of the extracellular matrix can stimulate calcium transients. This raises the possibility that the CD9 and CD24 glycoproteins identified as upregulated commonality genes may interact with these calcium buffering agents in a concerted mechanism to influence calcium levels that could in turn influence mossy fiber outgrowth.

Although compelling, the shifts in commonality gene function demonstrated in our analysis must still be regarded as trends because of the inherent difficulties in testing their statistical rigor. These difficulties are attributable primarily to (1) the derivation, and hence lack of independence, of the commonality gene group from the groups of development- and epileptogenesis-regulated genes, and (2) the lack of complete, uniform annotation of each of the roughly 7000 named genes on the Affymetrix microarray that we have used. This latter endeavor is ongoing, and in this direction we conducted an ANOVA analysis of 722 named genes on the microarray presently annotated into 15 functional gene clusters as defined by the GO database. Although not directly comparable with our functional analysis in Figure 3, this analysis was performed to provide a perspective of the statistical significance of the patterns of gene regulation evidenced in our microarray data. Despite the conservative nature of the ANOVA stemming from the potential counteraction of upregulated and downregulated genes within a cluster, our analysis identified 7 of 15 clusters as significantly regulated during development and/or epileptogenesis, including those containing genes related to defense/stress, calcium-mediated signaling, and morphology. Although these findings do not directly address the issue of commonality gene function, our ANOVA results in general support the likelihood of development- and epileptogenesis-associated regulation of some common functional groups of genes.

Although the analysis of our microarray data supports the hypothesis of parallels existing between DGC gene expression underlying developmental and epileptogenic plasticity, the question remains regarding whether we have identified one or more genes that are directly involved in DGC neurogenesis, mossy fiber outgrowth, or other shared aspects of development and epileptogenesis. Although the ultimate answer to this question will re- 
quire direct assessment of gene product function, our in situ descriptions of commonality gene expression have identified a number of promising candidates for further investigation. One such gene product of interest is thymosin $\beta-10$, a regulator of actin dynamics (Yu et al., 1993) normally expressed predominantly in immature DGCs, that appears to be preferentially induced in the more mature neurons of the DGC layer after SE. This pattern of upregulation during epileptogenesis resembles previous changes seen in expression of members of the bHLH gene family (Elliott et al., 2001) and further supports the possibility that older cells can reinitiate developmental patterns of gene expression after certain stimuli. Another upregulated commonality gene product, the cell-surface glycoprotein CD24, shows a profile of mRNA increase that is limited to the neurogenic SGZ and peaks at $7 \mathrm{~d}$ after SE, directly paralleling the profile of increased neurogenesis after SE (Parent et al., 1997). As a potential mediator of cell surface recognition and signaling during neuronal migration and axon outgrowth (Kleene et al., 2001), CD24 is an excellent candidate for guiding the integration of newborn neurons in the developing and epileptic dentate gyrus. Decreases in hippocalcin mRNA expression, which occur over a slower, slightly delayed timeframe, are seen in an almost linear gradient across the dentate gyrus, with the outer aspects of the DGC layer showing the largest decreases, perhaps reflecting an age-dependent ability (or lack thereof) to buffer intracellular calcium. Although DGCs are typically resistant to seizure-induced cell death, a similar gradient of intracellular calcium levels could have effects on more subtle calcium-mediated pathways such as the response of sprouting mossy fibers to guidance cues in the environment. In sum, the application of commonality screening appears to be a useful means to identify genes that may play important roles in DGC neurogenesis and axon outgrowth and may be a general strategy worth considering in other studies of gene expression.

The timepoints during development and epileptogenesis analyzed in this study were chosen to investigate gene expression at peak times of neurogenesis and axon outgrowth. However, we acknowledge that molecular factors that play a role in the initiation and perhaps termination of these events may have been missed. The markedly different functional composition of the large number of genes induced rapidly (i.e., within hours) after SE (Hevroni et al., 1998) supports this possibility and suggests that a more comprehensive microarray analysis across multiple timepoints during development and epileptogenesis is warranted. Indeed, several genes identified in this study as downregulated during development and upregulated during epileptogenesis, including PCTAIRE 2, SHARP-1, CPG2, and neuritin [the gene product of which has demonstrated neurite outgrowthpromoting activity (Naeve et al., 1997)], are highly expressed in post-mitotic neurons and hence are potential commonality genes at later timepoints. Nevertheless, our results indicate that what is presently known and what may be learned in the future regarding developmental changes in DGC gene expression and network establishment may provide important clues regarding molecular and cellular alterations associated with epileptogenesis, and vice versa. Similar observations regarding the cortical response to ischemic brain injury (Cramer and Chopp, 2000), as well as the recent evidence of increased neurogenesis in association with enriched environments and learning tasks (Kempermann et al., 1997; Gould et al., 1999a), suggest that further investigations into the potential reiteration of developmental molecular mechanisms in the adult brain during these processes may also be worthwhile.

\section{References}

Altman J, Bayer SA (1990) Migration and distribution of two populations of hippocampal granule cell precursors during the perinatal and postnatal periods. J Comp Neurol 301:365-381.

Altman J, Das GD (1965) Autoradiographic and histological evidence of postnatal hippocampal neurogenesis in rats. J Comp Neurol 124:319-335.

Baraban SC, Hollopeter G, Erickson JC, Schwartzkroin PA, Palmiter RD (1997) Knock-out mice reveal a critical antiepileptic role for neuropeptide Y. J Neurosci 17:8927-8936.

Bayer SA (1980) Development of the hippocampal region in the rat. I. Neurogenesis examined with ${ }^{3} \mathrm{H}$-thymidine autoradiography. J Comp Neurol 190:87-114.

Braunewell KH, Gundelfinger ED (1999) Intracellular neuronal calcium sensor proteins: a family of EF-hand calcium-binding proteins in search of a function. Cell Tissue Res 295:1-12.

Chang B, Somogyi R, Fuhrman S (2001) Evidence for shared genetic programs from cluster analysis of hippocampal gene expression dynamics in development and response to injury. Restor Neurol Neurosci 18:115-125.

Cramer SC, Chopp M (2000) Recovery recapitulates ontogeny. Trends Neurosci 23:265-271.

Cronin J, Dudek FE (1988) Chronic seizures and collateral sprouting of dentate mossy fibers after kainic acid treatment in rats. Brain Res 474:181-184.

Eisen MB, Spellman PT, Brown PO, Botstein D (1998) Cluster analysis and display of genome-wide expression patterns. Proc Natl Acad Sci USA 95:14863-14868.

Elliott RC, Khademi S, Pleasure SJ, Parent JM, Lowenstein DH (2001) Differential regulation of basic helix-loop-helix mRNAs in the dentate gyrus following status epilepticus. Neuroscience 106:79-88.

Eriksson PS, Perfilieva E, Bjork-Eriksson T, Alborn AM, Nordborg C, Peterson DA, Gage FH (1998) Neurogenesis in the adult human hippocampus. Nat Med 4:1313-1317.

Frotscher M, Zimmer J (1983) Lesion-induced mossy fibers to the molecular layer of the rat fascia dentata: identification of postsynaptic granule cells by the Golgi-EM technique. J Comp Neurol 215:299-311.

Gaubatz S, Meichle A, Eilers M (1994) An E-box element localized in the first intron mediates regulation of the prothymosin alpha gene by c-myc. Mol Cell Biol 14:3853-3862.

Ginzburg I, Teichman A, Dodemont HJ, Behar L, Littauer UZ (1985) Regulation of three beta-tubulin mRNAs during rat brain development. EMBO J 4:3667-3673.

Gomez TM, Robles E, Poo M, Spitzer NC (2001) Filopodial calcium transients promote substrate-dependent growth cone turning. Science 291:1983-1987.

Gould E, Tanapat P, Hastings NB, Shors TJ (1999a) Neurogenesis in adulthood: a possible role in learning. Trends Cogn Sci 3:186-192.

Gould E, Reeves AJ, Fallah M, Tanapat P, Gross CG, Fuchs E (1999b) Hippocampal neurogenesis in adult Old World primates. Proc Natl Acad Sci USA 96:5263-5267.

Hevroni D, Rattner A, Bundman M, Lederfein D, Gabarah A, Mangelus M, Silverman MA, Kedar H, Naor C, Kornuc M, Hanoch T, Seger R, Theill LE, Nedivi E, Richter-Levin G, Citri Y (1998) Hippocampal plasticity involves extensive gene induction and multiple cellular mechanisms. J Mol Neurosci 10:75-98.

Houser CR (1999) Neuronal loss and synaptic reorganization in temporal lobe epilepsy. Adv Neurol 79:743-761.

Kempermann G, Kuhn HG, Gage FH (1997) More hippocampal neurons in adult mice living in an enriched environment. Nature 386:493-495.

Kleene R, Yang H, Kutsche M, Schachner M (2001) The neural recognition molecule L1 is a sialic acid-binding lectin for CD24, which induces promotion and inhibition of neurite outgrowth. J Biol Chem 276:21656-21663.

Kowalski C, Micheau J, Corder R, Gaillard R, Conte-Devolx B (1992) Agerelated changes in cortico-releasing factor, somatostatin, neuropeptide $\mathrm{Y}$, methionine enkephalin and beta-endorphin in specific rat brain areas. Brain Res 582:38-46.

Kuhn H, Dickinson-Anson H, Gage F (1996) Neurogenesis in the dentate gyrus of the adult rat: age-related decrease of neuronal progenitor proliferation. J Neurosci 16:2027-2033.

Lee CK, Klopp RG, Weindruch R, Prolla TA (1999) Gene expression profile of aging and its retardation by caloric restriction. Science 285:1390-1393. 
Lowenstein DH (2001) Circuitry reorganization following seizures. In: Brain plasticity and epilepsy (Engel Jr J, Lowenstein DH, Moshe SL, Schwartzkroin PA, eds), pp 209-228. San Diego: Academic.

Lurton D, Cavalheiro EA (1997) Neuropeptide-Y immunoreactivity in the pilocarpine model of temporal lobe epilepsy. Exp Brain Res 116:186-190.

Middleton FA, Mirnics K, Pierri JN, Lewis DA, Levitt P (2002) Gene expression profiling reveals alterations of specific metabolic pathways in schizophrenia. J Neurosci 22:2718-2729.

Ming G, Henley J, Tessier-Lavigne M, Song H, Poo M (2001) Electrical activity modulates growth cone guidance by diffusible factors. Neuron 29:441-452.

Mirnics K, Middleton FA, Marquez A, Lewis DA, Levitt P (2000) Molecular characterization of schizophrenia viewed by microarray analysis of gene expression in prefrontal cortex. Neuron 28:53-67.

Mody M, Cao Y, Cui Z, Tay KY, Shyong A, Shimizu E, Pham K, Schultz P, Welsh D, Tsien JZ (2001) Genome-wide gene expression profiles of the developing mouse hippocampus. Proc Natl Acad Sci USA 98:8862-8867.

Naeve GS, Ramakrishnan M, Kramer R, Hevroni D, Citri Y, Theill LE (1997) Neuritin: a gene induced by neural activity and neurotrophins that promotes neuritogenesis. Proc Natl Acad Sci USA 94:2648-2653.

Okazaki MM, Evenson DA, Nadler JV (1995) Hippocampal mossy fiber sprouting and synapse formation after status epilepticus in rats: visualization after retrograde transport of biocytin. J Comp Neurol 352:515-534.

Parent J, Yu TW, Leibowitz RT, Geschwind DH, Sloviter RS, Lowenstein DH (1997) Dentate granule cell neurogenesis is increased by seizures and contributes to aberrant network plasticity in the adult hippocampus. J Neurosci 17:3727-3738.

Parent JM, Tada E, Fike JR, Lowenstein DH (1999) Inhibition of dentate granule cell neurogenesis with brain irradiation does not prevent seizureinduced mossy fiber synaptic reorganization in the rat. J Neurosci 19:4508-4519.

Pevny LH, Sockanathan S, Placzek M, Lovell-Badge R (1998) A role for SOX1 in neural determination. Development 125:1967-1978.

Pleasure SJ, Collins AE, Lowenstein DH (2000) Unique expression patterns of cell fate molecules delineate sequential stages of dentate gyrus development. J Neurosci 20:6095-6105.

Rampon C, Jiang CH, Dong H, Tang YP, Lockhart DJ, Schultz PG, Tsien JZ, $\mathrm{Hu} Y$ (2000) Effects of environmental enrichment on gene expression in the brain. Proc Natl Acad Sci USA 97:12880-12884.

Raza M, Pal S, Rafiq A, DeLorenzo RJ (2001) Long-term alteration of cal- cium homeostatic mechanisms in the pilocarpine model of temporal lobe epilepsy. Brain Res 903:1-12.

Represa A, Ben-Ari Y (1997) Molecular and cellular cascades in seizureinduced neosynapse formation. Adv Neurol 72:25-34.

Sandberg R, Yasuda R, Pankratz DG, Carter TA, Del Rio JA, Wodicka L, Mayford M, Lockhart DJ, Barlow C (2000) Regional and strain-specific gene expression mapping in the adult mouse brain. Proc Natl Acad Sci USA 97:11038-11043.

Shirasawa T, Akashi T, Sakamoto K, Takahashi H, Maruyama N, Hirokawa K (1993) Gene expression of CD24 core peptide molecule in developing brain and developing non-neural tissues. Dev Dyn 198:1-13.

Sloviter RS (1992) Possible functional consequences of synaptic reorganization in the dentate gyrus of kainate-treated rats. Neurosci Lett 137:91-96

Sloviter RS (1996) Hippocampal pathology and pathophysiology in temporal lobe epilepsy. Neurologia 11[Suppl 4]:29-32.

Tauck D, Nadler J (1985) Evidence of functional mossy fiber sprouting in hippocampal formation of kainic acid-treated rats. J Neurosci 5:1016-1022.

Thibault C, Lai C, Wilke N, Duong B, Olive MF, Rahman S, Dong H, Hodge CW, Lockhart DJ, Miles MF (2000) Expression profiling of neural cells reveals specific patterns of ethanol-responsive gene expression. Mol Pharmacol 58:1593-1600.

Tusher VG, Tibshirani R, Chu G (2001) Significance analysis of microarrays applied to the ionizing radiation response. Proc Natl Acad Sci USA 98:5116-5121.

Wegner M (1999) From head to toes: the multiple facets of Sox proteins. Nucleic Acids Res 27:1409-1420.

Woldbye DP, Larsen PJ, Mikkelsen JD, Klemp K, Madsen TM, Bolwig TG (1997) Powerful inhibition of kainic acid seizures by neuropeptide Y via Y5-like receptors. Nat Med 3:761-764.

Yu FX, Lin SC, Morrison-Bogorad M, Atkinson MA, Yin HL (1993) Thymosin beta 10 and thymosin beta 4 are both actin monomer sequestering proteins. J Biol Chem 268:502-509.

Zhang L, Wang L, Ravindranathan A, Miles MF (2002) A new algorithm for analysis of oligonucleotide arrays: application to expression profiling in mouse brain regions. J Mol Biol 317:225-235.

Zirlinger M, Kreiman G, Anderson DJ (2001) Amygdala-enriched genes identified by microarray technology are restricted to specific amygdaloid subnuclei. Proc Natl Acad Sci USA 98:5270-5275. 